\title{
SIMILARITY SOLUTIONS AND CONSERVATION LAWS FOR THE BEAM EQUATIONS: A COMPLETE STUDY
}

\section{Amlan Kanti Halder ${ }^{a, *}$, Andronikos Paliathanasis ${ }^{b, c}$, Peter Gavin LaWrence Leach ${ }^{c, d}$}

\author{
${ }^{a}$ Pondicherry University, Department of Mathematics, 605014 Kalapet, India \\ ${ }^{b}$ Universidad Austral de Chile, Instituto de Ciencias Físicas y Matemáticas, Valdivia, Chile \\ ${ }^{c}$ Durban University of Technology, Institute for Systems Science, Durban 4000, Republic of South Africa \\ ${ }^{d}$ University of KwaZulu-Natal, School of Mathematics, Statistics and Computer Science, Durban, South Africa \\ * corresponding author: amlan91.res@pondiuni.edu.in
}

\begin{abstract}
We study the similarity solutions and we determine the conservation laws of various forms of beam equations, such as Euler-Bernoulli, Rayleigh and Timoshenko-Prescott. The travelling-wave reduction leads to solvable fourth-order odes for all the forms. In addition, the reduction based on the scaling symmetry for the Euler-Bernoulli form leads to certain odes for which there exists zero symmetries. Therefore, we conduct the singularity analysis to ascertain the integrability. We study two reduced odes of second and third orders. The reduced second-order ode is a perturbed form of Painlevé-Ince equation, which is integrable and the third-order ode falls into the category of equations studied by Chazy, Bureau and Cosgrove. Moreover, we derived the symmetries and its corresponding reductions and conservation laws for the forced form of the abovementioned beam forms. The Lie Algebra is mentioned explicitly for all the cases.
\end{abstract}

KEYWORDS: Symmetry analysis, singularity analysis, conservation laws, beam equation.

\section{INTRODUCTION}

There are basically two types of beams. One type is supported at both ends and the other type is supported at only one end. It is a cantilever. The latter is of greater mathematical and physical interest for the free end can vibrate. This causes stresses in the beam. The first mathematical description was made by Leonhard Euler and Daniel Bernoulli around 1750, but there were some earlier attempts by Leonardo da Vinci and Galileo Galilei who were more than a little hampered by no knowledge of differential equations. Jacob Bernoulli laid the groundwork for the development of Leonhard Euler and Daniel Bernoulli. In 1894, the polymath Lord Rayleigh proposed an improvement to the Euler-Bernoulli model by including a term related to rotational stress. In 1921, Timoshenko introduced considerable improvements in what is now termed the Timoshenko-Prescott model.

There has been a considerable experimental and numerical work devoted to the comparison of predictions of the theories and experimental results. It should be emphasised that the infinitesimal theory of elasticity is three-dimensional and that the three models mentioned above are linear models. They make for easier mathematics, but there is a price to pay. Curiously, the simplest model, that of Euler-Bernoulli, still finds favour amongst some practitioners.

Some of the experimental work [1] undertaken to compare reality with theoretical prediction tries to make the experiment as close to a one-dimensional model as possible. One of the more interesting studies is the propagation of shock waves along the beam. This involves firing a bullet into the fixed end of the cantilever which is of a small diameter - $25 \mathrm{~mm}$ - to emulate a uniform boundary condition at the fixed end of the beam.

The literature devoted to the theory and practice of beams is extensive both in time and space. A fairly recent paper by Labuschagne [2] is very good in its historical aspects as well as being clearly written. Earlier papers in addition to that of Davies cited above are by Hudson [3 and Bancroft [4. An interesting feature is that the beams are taken to be cylindrical in shape even though the beams one sees in buildings are anything but cylindrical with some exceptions to be found in beamed structures of the nineteenth century. One assumes that this makes the analysis simpler due to the radial symmetry. Even a square cross-section would significantly complicate the mathematics. 
In this work, we study the algebraic properties of the Euler-Bernoulli, the Rayleigh and of the TimoshenkoPrescott according to the admitted Lie point symmetries, for the source-free equation as also in the case where a homogeneous source term exists. The application of the symmetry analysis for the Euler-Bernoulli equation is not new, there are various studies in the literature [5] 9], however in this paper, we obtained some new results, as the reduction of the Euler-Bernoulli form to a perturbed form of Painlevé-Ince 10 equation, which is integrable and the third-order ode, which falls into the category of equations studied by Chazy, Bureau and Cosgrove. Also, we show that the three beam equations of our study admit the same travelling-wave solution.

Certain phenomenal works were recently done for the static Euler-Bernoulli equation by Ruiz [11] and Da Silva PL [12. In Ruiz [11, the Euler-Bernoulli equation with an external agent is studied with respect to the joint invariants of the algebra and complete solutions are specified whereas in [12, for the static Euler-Bernoulli equation with specific nonlinear term, it was found that the algebraic structure of Lie point symmetries is similar to that of the Noether symmetries. It is worthwhile to mention the paper of Freire IL [13] where the Lane-Emden system is reduced to the Emden-Fowler equations and, correspondingly, the solutions of the system have been studied with the aid of the point symmetries. To elaborate the above mentioned works, we focus on the more general Euler-Bernoulli equation with and without the external forcing term and compute its solution using the point symmetries. It is also our intuition that the point symmetries of the form of Euler-Bernoulli under consideration do possess some similarities with the Noether symmetries to follow the results of the above mentioned work.

This paper is structured in the following way: In Section 2, we mention the Lie point symmetries and the corresponding algebra. In Section 3, we discuss the travelling-wave solutions for all the beam forms and further reductions of Euler-Bernoulli form using the scaling symmetries. In Section 4, we study the forced forms of the beam equations. Section 5 is devoted to the singularity analysis of a third-order equation, which is obtained by the reduction of Euler-Bernoulli equation by using the scaling symmetry. Conservation laws for the three beam equations are derived in Section 6. The conclusion and appropriate references are mentioned henceforth.

\section{LIE SYMMETRY ANALYSIS}

For the convenience of the reader, we give a briefly discussion in the theory of Lie point symmetries. In particular, we present the basic definitions and main steps for the determination of Lie point symmetries for a give differential equation. Consider $H^{A}\left(t, x, u, u_{, i}\right)=0$ to be a set of differential equations and $u_{, i}=\frac{\partial u}{\partial y^{i}}$ in which $y^{i}=(t, x)$.

Then, under the action of the infinitesimal one-parameter point transformation

$$
\begin{aligned}
t^{\prime} & =t(t, x, u ; \varepsilon), \\
x^{\prime} & =x(t, x, u ; \varepsilon), \\
u^{\prime} A & =u^{A}(t, x, u ; \varepsilon),
\end{aligned}
$$

in which $\varepsilon$ is an infinitesimal parameter, the set of differential equations $H^{A}$ is invariant if and only if,

$$
H^{A}\left(t^{\prime}, x^{\prime}, u^{\prime}\right)=H^{A}(t, x, u),
$$

or equivalently

$$
\lim _{\varepsilon \rightarrow 0} \frac{H^{A}\left(t^{\prime}, x^{\prime}, u^{\prime} ; \varepsilon\right)-H^{A}(t, x, u)}{\varepsilon}=0 .
$$

The latter expression is the definition of the Lie derivative $\mathcal{L}$ of $H^{A}$ along the direction

$$
\Gamma=\frac{\partial t^{\prime}}{\partial \varepsilon} \partial_{t}+\frac{\partial x^{\prime}}{\partial \varepsilon} \partial_{x}+\frac{\partial u^{\prime}}{\partial \varepsilon} \partial_{u}
$$

Hence, we shall say that the vector field $\Gamma$ will be a Lie point symmetry for the set of differential equations $H^{A}$ if and only if the following condition is true

$$
\mathcal{L}_{\Gamma}\left(H^{A}\right)=0 .
$$

In other words, the operator $\Gamma$ can be considered to be symmetry provided

$$
\Gamma^{[n]} H^{A}=0
$$


whenever $H^{A}\left(t, x, u, u,,_{i}\right)=0$ and $\Gamma^{[n]}$ denotes the $n$-th prolongation of the specified operator in its defined space. The set of all such operators can be denoted by $\mathbf{G}$, which can be regarded as the symmetry group for the set of differential equations $H^{A}\left(t, x, u, u,,_{i}\right)=0[14-16$.

\subsection{The Euler-Bernoulli Equation.}

The Euler-Bernoulli form of the beam equation is [1, 17,

$$
\alpha \beta u_{x x x x}+u_{t t}=0 .
$$

The Lie point symmetries are

$$
\begin{aligned}
& \Gamma_{1 a}=\partial_{x}, \Gamma_{2 a}=\partial_{t}, \\
& \Gamma_{3 a}=u \partial_{u}, \Gamma_{4 a}=2 t \partial_{t}+x \partial_{x}, \\
& \Gamma_{5 a}=a(t, x) \partial_{u}
\end{aligned}
$$

where $a(t, x)$ satisfies the Euler-Bernoulli form of the beam equation. The Lie Algebra is $\left(A_{3,3} \oplus A_{1}\right) \oplus_{s} \infty A_{1}$, according to the Morozov-Mubarakzyanov classificatin scheme [18 21].

\subsection{The Rayleigh equation.}

The Rayleigh form of the beam equation is [1, 17,

$$
\alpha \beta u_{x x x x}+u_{t t}-\beta u_{x x t t}=0 .
$$

The Lie point symmetries are

$$
\begin{aligned}
& \Gamma_{1 b}=\partial_{t}, \Gamma_{2 b}=\partial_{x}, \\
& \Gamma_{3 b}=u \partial_{u}, \Gamma_{4 b}=b(t, x) \partial_{u},
\end{aligned}
$$

where $b(t, x)$ satisfies the Rayleigh form of the beam equation. Consequently, the admitted Lie algebra is $A_{3} \oplus_{s} \infty A_{1}$.

\subsection{The Timoshenko-Prescott equation.}

The Timoshenko and Prescott form of the beam equation is [1, 24],

$$
\alpha \beta u_{x x x x}+u_{t t}-\beta(1+\epsilon) u_{x x t t}+\frac{\epsilon \beta u_{t t t t}}{\alpha}=0 .
$$

The Lie point symmetries are

$$
\begin{aligned}
& \Gamma_{1 c}=\partial_{t}, \Gamma_{2 c}=\partial_{x}, \Gamma_{3 c}=u \partial_{u} \\
& \Gamma_{4 c}=c(t, x) \partial_{u}
\end{aligned}
$$

where $c(t, x)$ satisfies the Timoshenko and Prescott form of the beam equation. Hence, the admitted Lie Algebra is $A_{3} \oplus_{s} \infty A_{1}$.

Therefore, we can say that the Timoshenko-Prescott equation and the Rayleigh equation are algebraic equivalent, but different with the Euler-Bernoulli equation as it admits a higher-dimensional Lie algebra.

We proceed our analysis by applying the Lie point symmetries to determine similarity solutions for the three equations of our study.

\footnotetext{
${ }^{1}$ We use the SYM package developed by Prof.Stelios Dimas [22, 23].
} 


\section{The TRAVElling-WAVE SOlution}

The travelling-wave solution for eq (8), with respect to $\Gamma_{2 a}+c \Gamma_{1 a}$, where $c$, denotes the frequency, leads to the fourth order equation,

$$
c^{2} v^{\prime \prime}(s)+\alpha \beta v^{\prime \prime \prime \prime}(s)=0
$$

where

$$
\begin{aligned}
s & =x-c t, \\
v(s) & =u(x, t) .
\end{aligned}
$$

The Lie point symmetries of equation 11 are

$$
\begin{aligned}
\Gamma_{1 d} & =\partial_{s}, \\
\Gamma_{2 d} & =\partial_{v}, \\
\Gamma_{3 d} & =s \partial_{v}, \\
\Gamma_{4 d} & =v \partial_{v}, \\
\Gamma_{5 d} & =\sin \frac{c s}{\sqrt{\alpha \beta}} \partial_{v}, \\
\Gamma_{6 d} & =\cos \frac{c s}{\sqrt{\alpha \beta}} \partial_{v} .
\end{aligned}
$$

The reduced form has six symmetries and hence linearisable, the solution for the fourth-order equation can be given as,

$$
v(s)=C_{0}+C_{1} s+C_{2} \sin \frac{c s}{\sqrt{\alpha \beta}}+C_{3} \cos \frac{c s}{\sqrt{\alpha \beta}},
$$

where $C_{i}, i=0,1,2,3$ are arbitrary constants. Corresponding, to which the solution for Euler-Bernoulli form of beam can be given as

$$
u(x, t)=C_{0}+C_{1}(x-c t)+C_{2} \sin \frac{c(x-c t)}{\sqrt{\alpha \beta}}+C_{3} \cos \frac{c(x-c t)}{\sqrt{\alpha \beta}} .
$$

\subsection{Further Reduction of the Euler-Bernoulli EQuATion.}

The reduction with respect to $\Gamma_{3 a}$ and $\Gamma_{4 a}$, leads to fourth-order odes. For the similarity variables with respect to $2 \Gamma_{3 a}+\Gamma_{4 a}$,

$$
\begin{aligned}
s & =\frac{t}{x^{2}}, \\
u(t, x) & =t v(s),
\end{aligned}
$$

the reduced ode is

$$
\left(\frac{2}{\alpha \beta}+120 s^{2}\right) v^{\prime}+\left(s+300 s^{3}\right) v^{\prime \prime}+144 s^{4} v^{\prime \prime \prime}+16 s^{5} v^{\prime \prime \prime \prime}=0 .
$$

The latter equation is solvable. We continue by considering the similarity variable $u(t, x)=x v(s)$, with respect to $\Gamma_{3 a}+\Gamma_{4 a}$ for $s$ being same as above leads to the fourth-order ode,

$$
24 s v^{\prime}+\left(\frac{1}{\alpha \beta}+156 s^{2}\right) v^{\prime \prime}+16 s^{3}\left(7 v^{\prime \prime \prime}+s v^{\prime \prime \prime \prime}\right)=0
$$

The equation has a total of five Lie point symmetries with $\partial_{v}, v \partial_{v}$ are the two simpler symmetries, the other three are in terms of Hypergeometric functions, which is complicated enough to be mentioned here.

We apply $\partial_{v}$ to perform the reduction. The new invariant functions are $s=h$ and $g(h)=v^{\prime}(s)$, hence, the reduced equation is a third-order ode,

$$
g^{\prime \prime \prime}(h)=-\frac{7 g^{\prime \prime}(h)}{h}-\left(\frac{39}{4 h^{2}}+\frac{1}{16 h^{4} \alpha \beta}\right) g^{\prime}(h)-\frac{3 g(h)}{2 h^{3}} .
$$


The latter equation admits four Lie point symmetries. The simpler one being $g \partial_{g}$, the other three are hyperbolic functions of $\sin h, \cos h$ and Hypergeometric function respectively. We consider $g \partial_{g}$, to do the reduction.

The subsequent second-order equation is,

$$
m^{\prime \prime}(n)=\left(-3 m(n)-\frac{7}{n}\right) m^{\prime}(n)-m(n)^{3}-\frac{7 m(n)^{2}}{n}-\left(\frac{39}{4 n^{2}}+\frac{1}{16 n^{4} \alpha \beta}\right) m(n)-\frac{3}{2 n^{3}},
$$

where $n=h$ and $m(n)=\frac{g^{\prime}(h)}{g(h)}$. This is the perturbed form of Painlevé-Ince equation and the singularity analysis of this equation shows that it is integrable. In our subsequent paper, we look at the analysis and discuss it elaborately. The reduction of $(12)$ with respect to $v \partial_{v}$, leads to a third-order equation with zero symmetries,

$$
\begin{aligned}
g^{\prime \prime \prime}(h)= & \left(-4 g(h)-\frac{7}{h}\right) g^{\prime \prime}(h)-3 g^{2}-\left(6 g(h)^{2}+21 \frac{g(h)}{h}+\frac{39}{4 h^{2}}+\frac{1}{16 h^{4} \alpha \beta}\right) g^{\prime}(h) \\
& -g(h)^{4}-\frac{7 g(h)^{3}}{h}-\left(\frac{39}{4 h^{2}}+\frac{1}{16 h^{4} \alpha \beta}\right) g(h)^{2}-\frac{3 g(h)}{2 h^{3}},
\end{aligned}
$$

where $h=s$ and $g(h)=\frac{v^{\prime}(s)}{v(s)}$. This equation is integrable as ascertained by the singularity analysis, the calculations of which are mentioned in a following section.

\subsection{The Travelling Wave solution for the Rayleigh EQUation.}

The reduction using $\Gamma_{1 b}+c \Gamma_{2 b}$ leads to the fourth-order equation which is maximally symmetric, where $c$ is the frequency. The definition of similarity variables $s$ and $v(s)$ is similar to that of the previous case.

$$
\left(1-\beta c^{2}\right) v^{\prime \prime \prime \prime}(s)+c^{2} v^{\prime \prime}(s)=0,
$$

which is in the form of equation (11).

\subsection{The travelling-Wave solution for the Timoshenko-Prescott equation.}

In a similar way, the application of the generic symmetry vector, $\Gamma_{1 c}+c \Gamma_{2 c}$, in 10 provides the fourth-order ode,

$$
\left(\alpha^{2} \beta-\beta c^{2} a-\alpha \beta c^{2} \varepsilon+c^{4} \varepsilon \beta\right) v^{\prime \prime \prime \prime}(s)+a c^{2} v^{\prime \prime}(s)=0,
$$

which is again in the form of (11). Consequently, we conclude that the three different beam equations provide the same travel-wave solutions.

We continue our analysis by assuming the existence of a source term $f(u)$ in the beam equations.

\section{SyMmetry ANALYSIS WITH A SOURCE TERM}

In this section, we study the impact of the forcing-source term $f(u)$ in the rhs of the Euler-Bernoulli, Rayleigh and Timoshenko-Prescott beam equations.

\subsection{Euler-Bernoulli.}

The Lie symmetry analysis for the Euler-Bernoulli equation 8 with the forced term $f(u)$, leads to the following possible cases for the forcing term

$$
\begin{gathered}
f_{1}(u)=a u+b, \\
f_{2}(u)=(a u+b)^{n}, \\
f_{3}(u)=e^{a u+b}, \\
f_{4}(u)=\text { arbitrary. }
\end{gathered}
$$

For $f_{1}(u)$, the admitted Lie point symmetries for the Euler-Bernoulli equation are,

$$
\Gamma_{1}^{f_{1}}=\partial_{t}, \Gamma_{2}^{f_{1}}=\partial_{x}, \Gamma_{3}^{f_{1}}=u \partial_{u}, \Gamma_{\infty}^{f_{1}}=b(t, x) \partial_{u}
$$


where they form the $3 A_{1}$ Lie Algebra and $b(t, x)$ is a solution of the original equation.

For the source $f_{2}(u)$, the admitted Lie point symmetries are,

$$
\Gamma_{1}^{f_{2}}=\partial_{t}, \Gamma_{2}^{f_{2}}=\partial_{x}, \Gamma_{3}^{f_{2}}=2(n-1) t \partial_{t}+(n-1) x \partial_{x}-4\left(u+\frac{b}{a}\right) \partial_{u},
$$

which they form the $2 A_{1} \oplus_{s} A_{1}$ Lie Algebra.

For $f_{3}(u)$ the admitted Lie point symmetries are,

$$
\Gamma_{1}^{f_{3}}=\partial_{t}, \Gamma_{2}^{f_{3}}=\partial_{x}, \Gamma_{3}^{f_{3}}=2 t \partial_{t}+x \partial_{x}-\frac{4}{a} \partial_{u},
$$

where the corresponding Lie Algebra is the $2 A_{1} \oplus_{s} A_{1}$.

Finally, for the arbitrary functional form of $f(u)$, the admitted Lie point symmetries are the only two symmetry vectors,

$$
\Gamma_{1}^{f_{4}}=\partial_{t}, \Gamma_{2}^{f_{4}}=\partial_{x},
$$

which form the $2 A_{1}$ Lie Algebra and provide the travelling-wave solution.

\subsection{Rayleigh and Timoshenko-Prescott eQuations.}

For the other two beam equations, namely the Rayleigh and Timoshenko-Prescott equations with a source term, we find that, for a linear function $f=f_{1}(u)$, the two equations admit the same Lie point symmetries with the force-free case, while for arbitrary function $f(u)=f_{4}(u)$, admit only two Lie point symmetries, the vector fields $\Gamma_{1}^{f_{4}}, \Gamma_{2}^{f_{4}}$ which provide travelling-wave solutions.

\subsection{Symmetry ClASSificAtion OF ODE.}

We show the reduction with the Lie point symmetries, $\Gamma_{1}^{f_{4}}+c \Gamma_{2}^{f_{4}}$, because the three beam equations of our consideration provide the same fourth-order ODE, which now, with a source term, takes the following form,

$$
v^{\prime \prime \prime \prime}+c^{2} v^{\prime \prime}=f(v),
$$

We perform the symmetry classification of the latter differential equation and we find that, for the arbitrary function $f(v)$, the latter equation admits only the autonomous symmetry vector $\partial_{v}$. However, for a constant source $f(v)=a_{0}$, the Lie point symmetries are,

$$
\partial_{s}, \partial_{v}, s \partial_{v},\left(2 c^{2} v-a_{0} s^{2}\right) \partial_{v}, \cos (c s) \partial_{v}, \sin (c s) \partial_{v},
$$

where the generic solution of equation $(22)$ is,

$$
v(s)=v_{1} \sin (c s)+v_{2} \cos (c s)+v_{3} s+v_{4}+\frac{a_{0}}{2 c^{2}} s^{2} .
$$

On the other hand, for $f(v)=a_{1} v+a_{0}$, equation 22 admits the six Lie point symmetries,

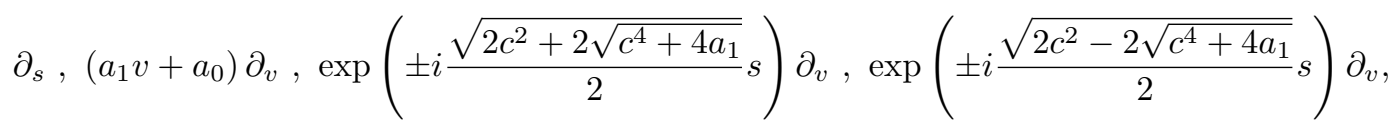

where the generic solution of $22 \mathrm{is}$,

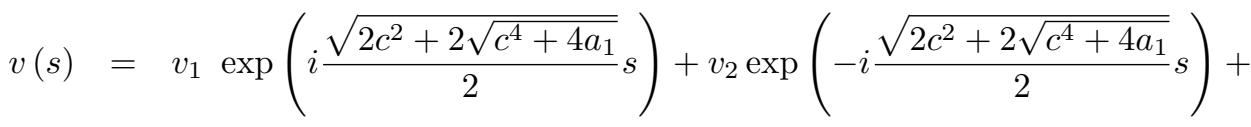

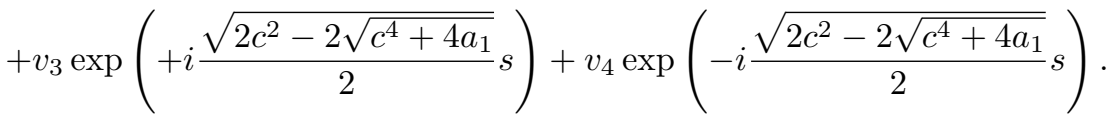




\subsection{SCAling SOlutions For the FORCED Euler-Bernoulli EQUATiOn.}

We continue by presenting the reduction with the scaling symmetries for the Euler-Bernoulli equation for the power-law and the exponential sources $f_{2}(u)$ and $f_{3}(u)$. For simplicity and without a loss of generality, we select $b=0$.

For the power-law source $f_{2}(u)=a u^{n}$, the application of the Lie point symmetry $\Gamma_{3}^{f_{2}}$ provides the reduced fourth-order ode,

$$
4 \alpha \beta v^{\prime \prime \prime \prime}+s^{2} v^{\prime \prime}+\frac{3 n+5}{n-1} s v^{\prime}+\frac{8(1+n) v}{(n-1)^{2}}-4 a v^{n}=0,
$$

in which $s=\frac{x}{\sqrt{t}}$ and $u(t, x)=v(s) t^{\frac{2}{n-1}}$.

For the exponential source $f_{3}(u)=e^{a u}$, the reduced equation given by the scaling symmetry is,

$$
4 \alpha \beta v^{\prime \prime \prime \prime}+s^{2} v^{\prime \prime}+3 s a v^{\prime}+4 a e^{a v}+8=0,
$$

where now $s=\frac{x}{\sqrt{t}}$ and $u(t, x)=-\frac{2}{a} \ln (t)+v(s)$.

\section{Singularity ANALYSIS}

The third-order ode that we apply the singularity analysis to is $15 p$ or

$$
\begin{aligned}
24 \nu x y(x)+y(x)^{2}+156 \nu x^{2} y(x)^{2}+112 \nu x^{3} y(x)^{3}+16 \nu x^{4} y(x)^{4}+y^{\prime}(x)+156 \nu x^{2} y^{\prime}(x) & \\
+336 \nu x^{3} y(x) y^{\prime}(x)++96 \nu x^{4} y(x)^{2} y^{\prime}(x)+48 \nu x^{4} y^{\prime 2}(x)+112 \nu x^{3} y^{\prime \prime}(x) & \\
+64 \nu x^{4} y(x) y^{\prime \prime}(x)+16 \nu x^{4} y^{\prime \prime \prime}(x) & =0,
\end{aligned}
$$

where $g(h)=y(x), h=x$ and $\alpha \beta=v$. We apply the ARS algorithm [25]27] and we make the usual substitution to obtain the leading-order behaviour [28,

$$
y \rightarrow a\left(x-x_{0}\right)^{p},
$$

which provides

$$
\begin{aligned}
32 a \nu p x^{4}\left(x-x_{0}\right)^{-3+p}-48 a \nu p^{2} x^{4}\left(x-x_{0}\right)^{-3+p}+16 a \nu p^{3} x^{4}\left(x-x_{0}\right)^{-3+p}-112 a \nu p x^{3}\left(x-x_{0}\right)^{-2+p} & \\
+112 a \nu p^{2} x^{3}\left(x-x_{0}\right)^{-2+p}+a p\left(x-x_{0}\right)^{-1+p}+156 a \nu p x^{2}\left(x-x_{0}\right)^{-1+p}+24 a \nu x\left(x-x_{0}\right)^{p} & \\
+a^{2}\left(x-x_{0}\right)^{2 p}+156 a^{2} \nu x^{2}\left(x-x_{0}\right)^{2 p}+112 a^{3} \nu x^{3}\left(x-x_{0}\right)^{3 p}+16 a^{4} \nu x^{4}\left(x-x_{0}\right)^{4 p} & \\
-64 a^{2} \nu p x^{4}\left(x-x_{0}\right)^{-2+2 p}+112 a^{2} \nu p^{2} x^{4}\left(x-x_{0}\right)^{-2+2 p}+336 a^{2} \nu p x^{3}\left(x-x_{0}\right)^{-1+2 p} & \\
+96 a^{3} \nu p x^{4}\left(x-x_{0}\right)^{-1+3 p} & =0 .
\end{aligned}
$$

From the latter, it is evident that $p \rightarrow-1$. Hence,

$$
\begin{array}{r}
-\frac{96 a \nu x^{4}}{\left(x-x_{0}\right)^{4}}+\frac{176 a^{2} \nu x^{4}}{\left(x-x_{0}\right)^{4}}-\frac{96 a^{3} \nu x^{4}}{\left(x-x_{0}\right)^{4}}+\frac{16 a^{4} \nu x^{4}}{\left(x-x_{0}\right)^{4}}+\frac{224 a \nu x^{3}}{\left(x-x_{0}\right)^{3}} \\
-\frac{336 a^{2} \nu x^{3}}{\left(x-x_{0}\right)^{3}}+\frac{112 a^{3} \nu x^{3}}{\left(x-x_{0}\right)^{3}}-\frac{a}{\left(x-x_{0}\right)^{2}} \\
+\frac{a^{2}}{\left(x-x_{0}\right)^{2}}-\frac{156 a \nu x^{2}}{\left(x-x_{0}\right)^{2}}+\frac{156 a^{2} \nu x^{2}}{\left(x-x_{0}\right)^{2}}+\frac{24 a \nu x}{x-x_{0}} .
\end{array}
$$

We extract the obvious dominant terms,

$$
-\frac{96 a \nu x^{4}}{\left(x-x_{0}\right)^{4}}+\frac{176 a^{2} \nu x^{4}}{\left(x-x_{0}\right)^{4}}-\frac{96 a^{3} \nu x^{4}}{\left(x-x_{0}\right)^{4}}+\frac{16 a^{4} \nu x^{4}}{\left(x-x_{0}\right)^{4}},
$$

and solve for $a$,

$$
(-3+a)(-2+a)(-1+a) a=0,
$$


to obtain,

$$
a \rightarrow 0, a \rightarrow 1, a \rightarrow 2, a \rightarrow 3 .
$$

In order to find the resonances, we substitute,

$$
y \rightarrow a\left(x-x_{0}\right)^{-1}+m\left(x-x_{0}\right)^{-1+s},
$$

and we linearize around $m$.

Then, the usual $x \rightarrow z+x_{0}$, simplify the calculations and provide the dominant terms factor as,

$$
16 \nu(-3+2 a+s)\left(2-6 a+2 a^{2}-3 s+2 a s+s^{2}\right) x_{0}^{4} z^{-4+s},
$$

and for the three values of $a$, we obtain the three sets of resonances, for each value of the coefficient term $a$ :

$$
\begin{aligned}
& a=1: s \rightarrow-1, s \rightarrow 1, s \rightarrow 2, \\
& a=2: s \rightarrow-2, s \rightarrow-1, s \rightarrow 1,
\end{aligned}
$$

and

$$
a=3: s \rightarrow-3, s \rightarrow-2, s \rightarrow-1 .
$$

For $a=1$, the solution is expressed in terms of a Right Painlevé Series, for $a=3$, in terms of a Left Painlevé Series and for $a=2$, in terms of a Mixed Painlevé Series.

We commence the consistency test. For the Right Painlevé Series, we write

$$
y \rightarrow\left(x-x_{0}\right)^{-1}+F_{0}+F_{1}\left(x-x_{0}\right)+F_{2}\left(x-x_{0}\right)^{2}+F_{3}\left(x-x_{0}\right)^{3}+\ldots
$$

where $F_{0}$ and $F_{1}$ are the second and third constants of integration. The output is enormous and hence omitted.

The substitution $x \rightarrow z+x_{0}$, just makes it easier to collect as powers. The terms in $z^{-1}$ are,

$$
\begin{aligned}
& \frac{2 F_{0}}{z}+\frac{24 \nu x_{0}}{z}+\frac{312 F_{0} \nu x_{0}^{2}}{z}+\frac{336 F_{0}^{2} \nu x_{0}^{3}}{z}+\frac{336 F_{1} \nu x_{0}^{3}}{z}+ \\
&+\frac{64 F_{0}^{3} \nu x_{0}^{4}}{z}+\frac{192 F_{0} F_{1} \nu x_{0}^{4}}{z}+\frac{128 F_{2} \nu x_{0}^{4}}{z}+\ldots \ldots=0 .
\end{aligned}
$$

This is solved to give,

$$
\begin{aligned}
64 \nu x_{0}^{4} F_{2} \rightarrow & -F_{0}-12 \nu x_{0}-156 F_{0} \nu x_{0}^{2}-168 F_{0}^{2} \nu x_{0}^{3}+ \\
& -168 F_{1} \nu x_{0}^{3}-32 a_{0}^{3} \nu x_{0}^{4}-96 F_{0} F_{1} \nu x_{0}^{4} .
\end{aligned}
$$

The expression for $F_{2}$ is substituted into the major output,

$$
\begin{aligned}
-7 F_{0}^{2}+3 F_{1}-240 \nu-\frac{22 F_{0}}{x_{0}}-2880 F_{0} \nu x_{0}-3780 F_{0}^{2} \nu x_{0}^{2}-2220 F_{1} \nu x_{0}^{2}+ \\
-1680 F_{0}^{3} \nu x_{0}^{3}-1680 F_{0} F_{1} \nu x_{0}^{3}-240 F_{0}^{4} \nu x_{0}^{4}+ \\
-480 F_{0}^{2} F_{1} \nu x_{0}^{4}+240 F_{1}^{2} \nu x_{0}^{4}+480 F_{3} \nu x_{0}^{4}=0,
\end{aligned}
$$

and the coefficient of the constant term is solved to give $F_{3}$ as,

$$
\begin{aligned}
480 \nu x_{0}^{5} F_{3}= & 22 F_{0}+7 F_{0}^{2} x_{0}-3 F_{1} x_{0}+240 \nu x_{0}+2880 F_{0} \nu x_{0}^{2}+3780 F_{0}^{2} \nu x_{0}^{3} \\
& +2220 F_{1} \nu x_{0}^{3}+1680 F_{0}^{3} \nu x_{0}^{4}+1680 F_{0} F_{1} \nu x_{0}^{4}+240 F_{0}^{4} \nu x_{0}^{5}+480 F_{0}^{2} F_{1} \nu x_{0}^{5}-240 F_{1}^{2} \nu x_{0}^{5} .
\end{aligned}
$$

Thus, there is not a problem with the determination of the coefficients of the terms in the Right Painlevé Series. As certain terms in the third-order ode are less dominant, there cannot be a Left Painlevé Series. The possibility of the existence of a Mixed Painlevé Series is moot due to the practical difficulty of calculating coefficients. Consequently, equation 15 is integrable according to the Painlevé test 


\section{Conservation laWs}

The Ibragimov's theory of nonlinear self-adjointness details the construction of conservation laws for a scalar pde 29 31. Our first step is to verify the self-adjointness condition on the various forms of beams and later on, compute the conservation laws. The preliminaries can be easily accessed from[29].

The main motivation behind using the Ibragimov's approach is to obtain the conservation laws to deduce certain special solutions for the Beam equations following the methodology specified by Cimpoiasu [32], where the author have used the nonlinear self-adjointness method to compute solutions for the Rossby waves. The Noether's theorem can be easily applied to obtain the conserved terms but it is our intuition that the non-local conserved terms as obtained using the Ibragimov's method can contribute in obtaining new solutions in a different subspace of the complex plane. The main objective is to deduce new solutions using the point symmetries, singularities and conservation laws. For instance, the Euler-Bernoulli equation does imply the existence of series type solutions through the method of Singularity analysis as mentioned in Section 5 for equation $(15)$.

Let the scalar PDE admit the following generators of the infinitesimal transformation,

$$
V=\xi^{i}\left(x, u, u_{i}, . .\right) \frac{\partial}{\partial x^{i}}+\eta\left(x, u, u_{i}, \ldots\right) \frac{\partial}{\partial u} .
$$

Then the scalar PDE and its adjoint equation, as defined above, admits the conservation law

$$
\begin{array}{r}
C^{i}=\xi^{i} L+W\left(\frac{\partial L}{\partial_{u_{i}}}-D_{j}\left(\frac{\partial L}{\partial_{u_{i j}}}\right)+D_{j} D_{k}\left(\frac{\partial L}{\partial_{u_{i j k}}}\right)-\ldots\right)+D_{j}(W) \\
\left(\frac{\partial L}{\partial_{u_{i j}}}-D_{k}\left(\frac{\partial L}{\partial_{u_{i j}}}\right)+\ldots\right) \\
+D_{j} D_{k}(W)\left(\frac{\partial L}{\partial_{u_{i j k}}}-\ldots\right),
\end{array}
$$

where $W=\eta-\xi^{i} u_{i}$ and $L$ denotes the Lagarangian of the corresponding form of the beam equation. For the Euler-Bernoulli, Rayleigh and Timshenko-Prescott forms the Lagarangians are as follows

$$
\begin{aligned}
& L=q(t, x)\left(u_{t t}+\alpha \beta u_{x x x x}\right), \\
& L=q(t, x)\left(\alpha \beta u_{x x x x}+u_{t t}-\beta u_{x x t t}\right), \\
& L=q(t, x)\left(\alpha \beta u_{x x x x}+u_{t t}-\beta(1+\epsilon) u_{x x t t}+\frac{\epsilon \beta u_{t t t t}}{\alpha}\right),
\end{aligned}
$$

where $q(t, x)$ is the new dependent variable. To verify the non-linear self adjointness, the substitution of $q(t, x)=\phi(t, x, u)$, to the adjoint equation of (8), (9) and (10) must satisfy for all solutions $u$ of those equations. The possible values of $\phi(t, x, u)$ is a constant term, let us say, $A_{0}$ and $A_{1} u(t, x)+A_{2}$, where $A_{1}$ and $A_{2}$ are arbitrary constants. A complete description of this method can be obtained from [29].

\subsection{Conservation laWs FOR the VARious Form of BeAm.}

With respect to each of the symmetries in (8), we compute the nonzero conservation laws.

For $\Gamma_{1 a}$, the conservation components are

$$
\begin{aligned}
c^{t} & =u_{x} \phi_{t}-u_{x t} \phi(t, x, u), \\
c^{x} & =\phi(t, x, u)\left(u_{t t}+\alpha \beta u_{x x x x}\right)+\alpha \beta u_{x} \phi_{x x x x}-\alpha \beta u_{x x} \phi_{x x}+\alpha \beta \phi_{x} u_{x x x}-u_{x x x x} \phi(t, x, u) .
\end{aligned}
$$

For $\Gamma_{2 a}$,

$$
\begin{aligned}
c^{t} & =\phi(t, x, u)\left(u_{t t}+\alpha \beta u_{x x x x}\right)+u_{t} \phi_{t}-u_{t t} \phi(t, x, u), \\
c^{x} & =\alpha \beta\left(u_{t} \phi_{x x x}-u_{x t} \phi_{x x}-u_{x x t} \phi_{x}-u_{x x x t} \phi(t, x, u)\right) .
\end{aligned}
$$


For $\Gamma_{3 a}$

$$
\begin{aligned}
c^{t} & =-u \phi_{t}+u_{t} \phi(t, x, u) \\
c^{x} & =\alpha \beta\left(u_{x} \phi_{x x}-u \phi_{x x x}+u_{x x} \phi_{x}+u_{x x x x}\right) .
\end{aligned}
$$

For $\Gamma_{4 a}$,

$$
\begin{aligned}
c^{t}= & 2 t\left(\phi(t, x, u)\left(u_{t t}+\alpha \beta u_{x x x x}\right)\right)+\phi_{t}\left(2 t u_{t}+x u_{x}\right)-\phi(t, x, u)\left(2 t u_{t t}+2 u_{t}+x u_{x t}\right), \\
c^{x}= & x\left(\phi(t, x, u)\left(u_{t t}+\alpha \beta u_{x x x x}\right)\right)+\alpha \beta \phi_{x x x}\left(2 t u_{t}+x u_{x}\right)-\alpha \beta \phi_{x x}\left(2 t u_{x t}+x u_{x x}+u_{x}\right) \\
& +\alpha \beta \phi_{x}\left(2 t u_{x x t}+x u_{x x x}+2 u_{x x}\right)-\alpha \beta \phi(t, x, u)\left(2 t u_{x x x t}+x u_{x x x x}+3 u_{x x x}\right) .
\end{aligned}
$$

F0r $\Gamma_{5 a}$

$$
\begin{aligned}
c^{t} & =-a(t, x) \phi_{t}+a_{t} \phi(t, x, u), \\
c^{x} & =-\alpha \beta a(t, x) \phi_{x x x}+\alpha \beta a_{x} \phi_{x x}-\alpha \beta a_{x x} \phi_{x}+\phi(t, x, u) a_{x x x} .
\end{aligned}
$$

Next, we compute the conservation laws of equation (9), with respect to its symmetries. $\Gamma_{1 b}$ leads to the following conserved components,

$$
\begin{aligned}
c^{t} & =\phi(t, x, u)\left(\alpha \beta u_{x x x x}+u_{t t}-\beta u_{x x t t}\right)+u_{t} \phi_{t}-\phi(t, x, u) u_{t t}, \\
c^{x} & =\alpha \beta\left(u_{t} \phi_{x x x}-u_{x t} \phi_{x x}+u_{x x t} \phi_{x}-u_{x x x t} \phi(t, x, u)\right) .
\end{aligned}
$$

For $\Gamma_{2 b}$,

$$
\begin{aligned}
c^{t} & =u_{x} \phi_{t}-u_{x t} \phi(t, x, u), \\
c^{x} & =\phi(t, x, u)\left(\alpha \beta u_{x x x x}+u_{t t}-\beta u_{x x t t}\right)+\alpha \beta\left(u_{x} \phi_{x x x}-u_{x x} \phi_{x x}+u_{x x x} \phi_{x}-\phi(t, x, u) u_{x x x x}\right) .
\end{aligned}
$$

For $\Gamma_{3 b}$,

$$
\begin{aligned}
c^{t} & =u_{t} \phi(t, x, u)-u \phi_{t}, \\
c^{x} & =\alpha \beta\left(u_{x} \phi_{x x}-u \phi_{x x x}-u_{x x} \phi_{x}+u_{x x x} \phi(t, x, u)\right) .
\end{aligned}
$$

For $\Gamma_{4 b}$

$$
\begin{aligned}
c^{t} & =b_{t} \phi(t, x, u)-b(t, x) \phi_{t}, \\
c^{x} & =\alpha \beta\left(b_{x} \phi_{x x}-b(t, x) \phi_{x x x}-b_{x x} \phi_{x}+b_{x x x} \phi(t, x, u)\right) .
\end{aligned}
$$

For the Timoshenko-Prescott form of the beam $10 p$, the conserved components are as follows: For $\Gamma_{1 c}$,

$$
\begin{aligned}
c^{t}= & \phi(t, x, u)\left(\alpha \beta u_{x x x x}+u_{t t}-\beta(1+\epsilon) u_{x x t t}+\frac{\epsilon \beta u_{t t t t}}{\alpha}\right)+u_{t}\left(\phi_{t}+\frac{\epsilon \beta \phi_{t t t}}{\alpha}\right)-u_{t t}\left(\phi(t, x, u)+\frac{\epsilon \beta \phi_{t t}}{\alpha}\right) \\
& +u_{t t t} \frac{\epsilon \beta \phi_{t}}{\alpha}-u_{t t t t} \frac{\epsilon \beta \phi(t, x, u)}{\alpha}, \\
c^{x}= & \alpha \beta\left(u_{t} \phi_{x x x}-u_{x t} \phi_{x x}+u_{x x t} \phi_{x}-u_{x x x t} \phi(t, x, u)\right) .
\end{aligned}
$$


For $\Gamma_{2 c}$,

$$
\begin{aligned}
c^{t}= & u_{x}\left(\phi_{t}+\frac{\phi_{t t t} \epsilon \beta}{\alpha}\right)-u_{x t}\left(\phi(t, x, u)+\frac{\phi_{t t} \epsilon \beta}{\alpha}\right)+u_{x t t} \frac{\phi_{t} \epsilon \beta}{\alpha}-u_{x t t t} \frac{\epsilon \beta}{\alpha} \\
c^{x}= & \phi(t, x, u)\left(\alpha \beta u_{x x x x}+u_{t t}-\beta(1+\epsilon) u_{x x t t}+\frac{\epsilon \beta u_{t t t t}}{\alpha}\right) \\
& +\alpha \beta\left(u_{x} \phi_{x x x}-u_{x x} \phi_{x x}+u_{x x x} \phi_{x}-\phi(t, x, u) u_{x x x x}\right) .
\end{aligned}
$$

For $\Gamma_{3 c}$

$$
\begin{aligned}
c^{t} & =-u\left(\phi_{t}+\frac{\epsilon \beta \phi_{t t t}}{\alpha}\right)+u_{t}\left(\phi(t, x, u)+\frac{\epsilon \beta \phi_{t t}}{\alpha}\right)-u_{t t} \frac{\epsilon \beta \phi_{t}}{\alpha}+u_{t t t} \frac{\epsilon \beta \phi(t, x, u)}{\alpha}, \\
c^{x} & =\alpha \beta\left(u_{x} \phi_{x x}-u \phi_{x x x}-u_{x x} \phi_{x}+u_{x x x} \phi(t, x, u)\right) .
\end{aligned}
$$

For $\Gamma_{4 c}$,

$$
\begin{aligned}
& c^{t}=-c(t, x)\left(\phi_{t}+\frac{\epsilon \beta \phi_{t t t}}{\alpha}\right)+c_{t}\left(\phi(t, x, u)+\frac{\epsilon \beta \phi_{t t}}{\alpha}\right)-c_{t t} \frac{\epsilon \beta \phi_{t}}{\alpha}+c_{t t t} \frac{\epsilon \beta \phi(t, x, u)}{\alpha}, \\
& c^{x}=\alpha \beta\left(c_{x} \phi_{x x}-c(t, x) \phi_{x x x}-c_{x x} \phi_{x}+c_{x x x} \phi(t, x, u)\right) .
\end{aligned}
$$

\section{Conclusion}

In this work, we focused on the algebraic properties for three different forms of the beam equations with or without a source. For the source free equations, we found that the Euler-Bernoulli equation is invariant under the Lie algebra $\left(A_{3,3} \oplus A_{1}\right) \oplus_{s} \infty A_{1}$, while, the Rayleigh and Timoshenko-Prescott equations are invariant under the Lie algebra $A_{3} \oplus_{s} \infty A_{1}$.

In the case of an isotropic source $f(u)$, we found that the Euler-Bernoulli, Rayleigh and Timoshenko-Prescott equations are invariant under the Lie algebra $2 A_{1}$ for an arbitrary source $f(u)$. Moreover, for the Euler-Bernoulli beam equations, the admitted Lie algebras are $A_{3} \oplus_{s} \infty A_{1}$ for Linear $f(u)=a u+b, 2 A_{1} \oplus_{s} A_{1}$ for exponential or power-law functional form. For the other two beam equations, there are no specific functional forms of $f(u)$, where the equations admit different algebras. Therefore, for the source-free equations, we derived the conservation laws by applying the Ibragimov's method.

We applied the Lie point symmetries to reduce the pdes and we proved that the three beam equations provide exactly the same travelling-wave solutions. The most important result of our paper is the reduction of Euler-Bernoulli equation to a second-order equation, of the form of perturbed Painlevé-Ince equation and to a third-order equation, which was studied by Chazy, Bureau and Cosgrove. One of our subsequent papers will be on the singularity analysis of the perturbed Painlevé-Ince equation. Moreover, our future work also includes deriving further solutions of the different forms of beams using conservation laws.

\section{ACKNOWLEDGEMENTS}

AKH expresses grateful thanks to UGC (India) NFSC, Award No. F1-17.1/201718/RGNF-2017-18-SC-ORI-39488 for financial support and late Prof. K.M.Tamizhmani for the discussions that formed the basis of this work. PGLL acknowledges the support of the National Research Foundation of South Africa, the University of KwaZulu-Natal and the Durban University of Technology and thanks the Department of Mathematics, Pondicherry University, for gracious hospitality.

\section{REFERENCES}

[1] R. M. Davies, G. I. Taylor. A critical study of the hopkinson pressure bar. Philosophical Transactions of the Royal Society of London Series A, Mathematical and Physical Sciences 240(821):375 - 457, 1948. DOI:10.1098/rsta.1948.0001

[2] A. Labuschagne, N. van Rensburg, A. van der Merwe. Comparison of linear beam theories. Mathematical and Computer Modelling 49(1):20 - 30, 2009. DOI:10.1016/j.mcm.2008.06.006 
[3] G. E. Hudson. Dispersion of elastic waves in solid circular cylinders. Physical Review 63:46 - 51, 1943. DOI:10.1103/PhysRev.63.46

[4] D. Bancroft. The velocity of longitudinal waves in cylindrical bars. Physical Review 59:588 - 593, 1941. DOI:10.1103/PhysRev.59.588

[5] A. H. Bokhari, F. M. Mahomed, F. D. Zaman. Symmetries and integrability of a fourth-order Euler-Bernoulli beam equation. Journal of Mathematical Physics 51(5):053517, 2010. DOI:10.1063/1.3377045

[6] A. Fatima, F. M. Mahomed, C. M. Khalique. Noether symmetries and exact solutions of an Euler-Bernoulli beam model. International Journal of Modern Physics B 30(28-29):1640011, 2016. DOI:10.1142/S0217979216400117

[7] D. Huang, X. Li, S. Yu. Lie symmetry classification of the generalized nonlinear beam equation. Symmetry 9:115, 2017. DOI:10.3390/sym9070115

[8] C. Wafo Soh. Euler-bernoulli beams from a symmetry standpoint-characterization of equivalent equations. Journal of Mathematical Analysis and Applications 345(1):387 - 395, 2008. DOI:10.1016/j.jmaa.2008.04.023.

[9] M. Tuz. The existence of symmetric positive solutions of fourth-order elastic beam equations. Symmetry 11:121, 2019. DOI:10.3390/sym11010121.

[10] E. L. Ince. Ordinary Differential Equations. Longmans Green \& Co, London, 1927.

[11] A. Ruiz, C. Muriel, J. Ramirez. Exact general solution and first integrals of a remarkable static Euler-Bernoulli beam equation. Communications in Nonlinear Science and Numerical Simulation 69:261 - 169, 2018. DOI:10.1016/j.cnsns.2018.09.012

[12] P. da Silva, I. Freire. Symmetry analysis of a class of autonomous even-order ordinary differential equations. IMA Journal of Applied Mathematics 80:1739 - 1758, 2015. DOI:10.1093/imamat/hxv014.

[13] I. L. Freire, P. L. da Silva, M. Torrisi. Lie and noether symmetries for a class of fourth-order emden-fowler equations. Journal of Physics A: Mathematical and Theoretical 46(24):245206, 2013. DOI:10.1088/1751-8113/46/24/245206

[14] W. Bluman, S. Kumei. Symmetries and Differential Equations. Springer, New York, 1989. DOI:10.1007/978-1-4757-4307-4

[15] Y. N. Grigoriev, V. F. Kovalev, S. Meleshko, N. Ibragimov. Symmetries of integro-differential equations: with applications in mechanics and plasma physics. Springer Dordrecht, 2010.

[16] P. J. Olver. Applications of Lie groups to differential equations. Springer Science \& Business Media, New York, 2000.

[17] J. M. Gere, S. P. Timoshenko. Mechanics of materials. PWS-KENT Publishing Company, 1997.

[18] V. V. Morozov. Classification of six-dimensional nilpotent Lie algebras. Izvestia Vysshikh Uchebn Zavendeniı Matematika 5:161 - 171, 1958.

[19] G. M. Mubarakzyanov. On solvable lie algebras. Izvestia Vysshikh Uchebn Zavendenǐ Matematika 34:99 - 106, 1963.

[20] G. M. Mubarakzyanov. Classification of real structures of five-dimensional lie algebras. Izvestia Vysshikh Uchebn Zavendenǐ Matematika 32:114 - 123, 1963.

[21] G. M. Mubarakzyanov. Classification of solvable six-dimensional lie algebras with one nilpotent base element. Izvestia Vysshikh Uchebn Zavendenǐ Matematika 35:104 - 116, 1963.

[22] S. Dimas, D. Tsoubelis. SYM: A new symmetry-finding package for Mathematica. Group Analysis of Differential Equations pp. 64 - 70, 2004.

[23] S. Dimas, D. Tsoubelis. A new mathematica-based program for solving overdetermined systems of pdes. In In 8th International Mathematica Symposium. 2006.

[24] W. T. Thomson, M. D. Dahleh. Theory of Vibration With Applications. Prentice-Hall, New Jersey, 1981.

[25] M. Ablowitz, A. Ramani, H. Segur. Nonlinear evolution equations and ordinary differential equations of painleve'type. Lettere al Nuovo Cimento 23(9):333 - 338, 1978. DOI:10.1007/BF02824479.

[26] M. J. Ablowitz, A. Ramani, H. Segur. A connection between nonlinear evolution equations and ordinary differential equations of P-type. I. Journal of Mathematical Physics 21(4):715 - 721, 1980. DOI:10.1063/1.524491

[27] M. J. Ablowitz, A. Ramani, H. Segur. A connection between nonlinear evolution equations and ordinary differential equations of P-type. II. Journal of Mathematical Physics 21(5):1006 - 1015, 1980. DOI:10.1063/1.524548.

[28] A. Paliathanasis, P. G. L. Leach. Nonlinear ordinary differential equations: A discussion on symmetries and singularities. International Journal of Geometric Methods in Modern Physics 13(07):1630009, 2016. DOI:10.1142/S0219887816300099 
[29] N. H. Ibragimov. Nonlinear self-adjointness and conservation laws. Journal of Physics A: Mathematical and Theoretical 44(43):432002, 2011. DOI:10.1088/1751-8113/44/43/432002.

[30] N. H. Ibragimov. A new conservation theorem. Journal of Mathematical Analysis and Applications 333(1):311 328, 2007. DOI:10.1016/j.jmaa.2006.10.078

[31] R. Tracina, M. Bruzon, M. L. Gandarias, M. Torrisi. Nonlinear self-adjointness, conservation laws, exact solutions of a system of dispersive evolution equations. Communications in Nonlinear Science and Numerical Simulation 19:3036 - 3043, 2014. DOI:10.1016/j.cnsns.2013.12.005

[32] R. Cimpoiasu, R. Constantinescu. Nonlinear self-adjointness and invariant solutions of a 2D Rossby wave equation. Central European Journal of Physics 12:81 - 89, 2014. DOI:10.2478/s11534-014-0430-6 